\title{
MADURACIÓN BIOLÓGICA DE PATINADORES ENTRE 8 Y 16 AÑOS DE EDAD DE LAS CIUDADES DE PEREIRA Y CARTAGO
}

Natalia García Zapata*, Alejandra Sánchez García*, Laura Marcela Rodas Domínguez $^{*}$, Alejandro Gómez Rodas**

\section{Resumen}

Introducción: el patinaje de velocidad es un deporte de iniciación temprana que clasifica categorías según edad cronológica, provocando que competencias y entrenamientos no estén acordes con el desarrollo biológico. El objetivo de esta investigación será determinar las características de la maduración biológica en patinadores entre los 8 y 16 años de edad de las ciudades de Pereira y Cartago.

Métodos: estudio cuantitativo observacional analítico con muestra no probabilística intencionada de 60 patinadores. Se realizará predicción de velocidad pico de crecimiento mediante ecuación de regresión lineal propuesta por Mirwald y colaboradores a partir de medidas antropométricas.

Resultados esperados: conocimiento acerca de procesos de crecimiento y desarrollo biológico en patinadores de 8-16 años y establecimiento de baremos comparativos con otras poblaciones.

Impactos esperados: mejoramiento y adecuación de la dosificación de las intensidades, volúmenes y densidades de entrenamiento acordes con los procesos de crecimiento y desarrollo de deportistas en formación.

* Investigación en curso.

* Estudiantes de V semestre de Fisioterapia. Fundación Universitaria del Área Andina, seccional Pereira. Integrantes del semillero Semfis.

* Docente Fundación Universitaria del Área Andina y Universidad Tecnológica de Pereira, Programas de Fisioterapia y Ciencias del Deporte y la Recreación. 


\section{Introducción}

El patinaje de velocidad es un deporte de iniciación temprana que utiliza la clasificación por edad cronológica para el diseño, programación de entrenamientos y competencias. Este hecho provoca que la dosis de intensidad, volumen y densidad en esta actividad no considere la individualización y especificidad del entrenamiento acorde con los procesos de crecimiento y desarrollo propios de la infancia y la adolescencia (1). Durante estos periodos vitales, la tasa de crecimiento y desarrollo fluctúa individualmente por factores genéticos, nutricionales y ambientales, hecho que se ve reflejado en la poca relación existente entre la edad cronológica y la maduración biológica $(1,2)$; lo que conduce a errores frecuentes en la prescripción de la cargas de entrenamiento en estas edades, facilitando la aparición de patologías músculo-esqueléticas asociadas al crecimiento como el Osgood-Schlatter, Sinding-Larsen-Johansson y enfermedad de Sever (3), como también la manifestación de síndrome por sobreentrenamiento y deserción temprana en deportistas (4). Por estas razones, el conocimiento del momento de maduración biológica en la infancia y adolescencia es de vital importancia para la adecuada planificación y programación de entrenamientos en deportes de iniciación temprana, hecho que ajustaría correctamente los procesos de adaptación biológica a las demandas de entrenamiento (1), permitiendo el sano crecimiento y desarrollo propiciado por la práctica deportiva, fomentando la promoción de la salud y la prevención de enfermedades músculo-esqueléticas y las capacidades de adaptación de estos organismos en proceso de desarrollo a las demandas específicas del entrenamiento.

Existe poca evidencia en torno a las características y manifestaciones de los procesos de maduración biológica en deportes de iniciación temprana dados los costos, características invasivas, experticia y aspectos éticos relativos a las valoraciones clínicas realizadas para conocer el estado de maduración biológica en niños y adolescentes $(5,6)$. Por esta razón, la utilización de sistemas de evaluación no invasivos basados en características antropométricas se convierten en alternativas costo-efectivas para la realización de este tipo de valoraciones en niños y adolescentes, especialmente en el ámbito deportivo.

Un aspecto determinante en las investigaciones con niños deportistas lo constituye el estudio de los niveles de maduración biológica, los cuales clasifican el desarrollo biológico en adelantado, promedio y tardío (5), ya que las variaciones que se encuentran en esta, afectan la capacidad motora y funcional, expresada en valencias físicas susceptibles de ser valoradas a partir de pruebas generales y específicas de aptitud física (5). La evaluación física y fisiológica del atleta se realizan, a menudo, en varias etapas durante un ciclo anual de formación para establecer la eficacia de bloques individuales de entrenamiento o generales del plan anual de formación. Sin embargo, al trabajar con niños o adolescentes tales cambios en el desempeño pueden verse significativamente afectados por el crecimiento y la maduración biológica, estos pueden afectar el rendimiento físico 
en la juventud o niñez del deportista (7). Investigadores y clínicos pediátricos han observado la importancia de considerar la maduración biológica para desarrollar programas de capacitación apropiados para optimizar la adaptación y así minimizar el riesgo de lesiones relacionadas con la actividad.

Ahora bien, aunque la edad cronológica es la forma tradicional de clasificar categorías competitivas en diversos deportes como también la forma de agrupar a los deportistas en formación para la dosificación y planificación del entrenamiento, es importante reconocer que la edad cronológica no siempre coincide con las características morfológicas y funcionales que van acorde con la maduración biológica del cuerpo. Por ejemplo, se pueden encontrar niños de 8-9 años cuyas características morfológicas y funcionales pueden corresponder a un niño de mayor edad, y viceversa (1); lo que significa que su rendimiento morfológico y funcional estará condicionado por el adelanto o atraso en el momento pico de crecimiento, evidenciándose grandes variaciones en el rendimiento deportivo en estas edades que no se podrían llegar a explicar desde la categorización por edad cronológica (4).

Si los entrenamientos y las competencias no son adaptadas a la maduración biológica de los niños, podemos encontrar diferencias en el rendimiento que van a afectar tanto la prescripción o dosificación de la carga o la respuesta del organismo de estos niños a la misma (8); con consecuencias inevitables como una alta probabilidad de sufrir síndromes de sobreentrenamiento en el peor de los casos o, por el contrario, que el estímulo recibido sea inferior a su condición biológica y potencial adaptativo conduciendo a una insuficiencia del entrenamiento para producir los mejores desarrollos fisiológicos y corporales en estos niños y adolescentes. En consecuencia, se produce una alta frecuencia de abandono deportivo en este deporte.

Así las cosas, es necesario indagar por las características de la maduración biológica en estos deportistas, dado que permitirá una adecuación óptima de las cargas de entrenamiento acordes con los procesos de desarrollo biológico y no al concepto de edad cronológica que podría no adaptarse muy bien a las prescripciones y dosificaciones del entrenamiento en estos niños (1). Esto llevará finalmente a que la adaptación corporal y fisiológica de estos niños a los entrenamientos, sea mucho mejor, potenciando sus capacidades y habilidades motrices, favoreciendo procesos de entrenamiento de iniciación temprana adaptados al desarrollo biológico progresivo de estos niños; lo que llevará finalmente a una mayor probabilidad de éxito deportivo y una prevención del abandono anticipado de este deporte (6). Por todas estas razones, el objetivo de la presente investigación será determinar las características de la maduración biológica en patinadores entre los 8 y 16 años de las ciudades de Pereira y Cartago.

\section{Materiales y métodos}

Metodología: tipo de estudio. Investigación con enfoque cuantitativo de tipo observacional analítico. 
Población: patinadores de género masculino y femenino en etapas de niñez mediana, preadolescencia y adolescencia de clubes de patinaje de Pereira y Cartago.

Tipo de muestreo: no probabilístico intencionado.

Muestra: estará constituida por 60 deportistas del género masculino y femenino distribuidos equitativamente por edad desde los 8 a los 16 años pertenecientes a clubes deportivos de patinaje de las ciudades de Pereira y Cartago. Los acudientes de los deportistas deberán firmar consentimiento informado de participación en la investigación y los deportistas asentimiento informado. Su participación estará sujeta a los lineamientos éticos de la declaración de Helsinki de investigación en seres humanos. Criterios de inclusión: estar inscrito a los clubes de patinaje "Ciudad del Sol", "Guepardos" y "Fénix perla del Otún”, tener una edad comprendida entre los 8 y 16 años de edad y firmar el consentimiento informado indicando su voluntad explícita de participación en la investigación.

Criterios de exclusión: antecedentes de fracturas que puedan alterar las mediciones antropométricas de longitudes y diámetros óseos, patologías asociadas a alteraciones del crecimiento y desarrollo y trastornos genéticos como el síndrome de Turner, síndrome de Down y la acondroplasia.

Variables: edad, género, estrato social económico y meses de entrenamiento, talla bípeda, talla sedente, longitud de miembros inferiores y peso corporal.
Técnicas e instrumentos para la recolección de los datos: se realizará una predicción mediante ecuación de regresión lineal múltiple propuesta por Mirwald y colaboradores $(1,5)$ de la velocidad pico de crecimiento a partir de medidas antropométricas que incluyen la talla bípeda, la talla sedente, el peso corporal, la longitud de miembros inferiores y la edad cronológica. El modelo de regresión lineal propuesto por Mirwald y colaboradores propone que estas variables predicen el $92 \%$ de la variabilidad en la velocidad pico de crecimiento con un R2 ajustado con valor de 0,92 y un error de estimación estándar para la velocidad pico de crecimiento correspondiente a 0,49 años. Una vez finalice la recolección de datos, se realizará la fase de análisis descriptivo inicial de variables sociodemográficas y antropométricas, seguida de una segunda fase de análisis bivariado comparativo para establecer diferencias y relaciones entre las variables de estudio. Para el análisis de datos se utilizará el paquete estadístico SPSS versión 24 licenciado por la Fundación Universitaria del Área Andina.

\section{Referencias}

1. Gómez-Campos R, De Arruda M, Hobold E, Abella CP, Camargo C, Martínez Salazar $\mathrm{C}$, et al. Valoración de la maduración biológica: usos y aplicaciones en el ámbito escolar. Rev Andaluza Med del Deport [Internet]. 2013; 6(4):151-160. http://dx. doi.org/10.1016/S1888-7546(13)70051-0

2. Cossio-Bolaños MA, Viveros-Flores $A$, Castillo-Retamal M, Vargas-Vitoria R, Gatica P, Gómez-Campos R. Patrones de actividad física en adolescentes en función del sexo, edad cronológica y biológica. Nutr Clin y Diet Hosp. 2015; 35(2):41-47. 
3. Pose LG. Lesiones deportivas osteocartilaginosas en el niño y adolescente. Rev Chil Radiol. 2005; 11(2):91-100.

4. Verdugo MF. El proceso de maduración biológica y el rendimiento deportivo. Rev Chil Pediatría. 2015; 86(6):383-385.

5. Malina RM, Rogol AD, Cumming SP, Coelho E, Silva MJ, Figueiredo AJ. Biological maturation of youth athletes: Assessment and implications. $\mathrm{Br} \mathrm{J}$ Sports Med. 2015; 49(13):852-859.

6. Navarro Valdivielso F. Entrenamiento adaptado a los jóvenes. Rev Educ. 2004; (335):61-80.
7. Rhodris L, Jon O, Avery F, Gregory M, Mark DSC. Chronological age vs. biological maduration: Inplicatons for exercise programming in youth. J Strength Cond Res. 2014; 1454-1464.

8. Dayse B, Marco F. Características morfológicas, motoras y de condición física de los niños escolares entre 6-12 años del municipio de Cerrito-Valle como criterio para la selección deportiva. Universidad del Valle; 2011. 